EPJ Web of Conferences 31, 00028 (2012)

DOI: $10.1051 /$ epjconf/20123100028

(C) Owned by the authors, published by EDP Sciences - SIF, 2012

\title{
Neutron and charged particle identification by means of various detectors
}

\author{
M. Sénoville ${ }^{1}$, N.L. Achouri ${ }^{1}$, F. Delaunay ${ }^{1}$, J. Gibelin ${ }^{1}$, \\ B. Laurent ${ }^{2}$, X. LedouX ${ }^{2}$, F.M. Marqués ${ }^{1}$, N.A. OrR ${ }^{1}$, \\ C. Fontbonne ${ }^{1}$, B. Carniol $^{1}$, T. Chaventré ${ }^{1}$, D. Cussol ${ }^{1}$, \\ D. Etasse ${ }^{1}$, J.M. Fontbonne ${ }^{1}$, J. HARAnG ${ }^{1}$, J. Hommet ${ }^{1}$, \\ H. Plard ${ }^{1}$, J. Poincheval ${ }^{1}$, \\ O. Lopez ${ }^{1}$, N. Le NeIndre ${ }^{1}$, B. Borderie ${ }^{3}$, M.F. Rivet ${ }^{3}$, \\ L. Tassan-Got ${ }^{3}$, P. PawŁowski ${ }^{4}$, G. TĂBĂCARU ${ }^{5}$, \\ A. Brauning-Demian ${ }^{6}$, J. Perronnel ${ }^{1}$, E. Balanzat ${ }^{7}$, \\ H. Hamrita ${ }^{7}$, G. Randisi ${ }^{1}$, D. Dumitriu ${ }^{9}$, D. Flueraşu ${ }^{9}$, \\ Y. ThimONT ${ }^{10}$, and M. PÂRLOG ${ }^{1,9}$ \\ and the INDRA and FAZIA COLLABORATIONS
}

\footnotetext{
${ }^{1}$ LPC, ENSICAEN, Université de Caen, CNRS/IN2P3, Caen, France ${ }^{2}$ CEA, DAM, DIF, 91297 Arpajon, France

${ }^{3}$ Institut de Physique Nucléaire, CNRS/IN2P3,Université Paris-Sud 11, F-91406 Orsay Cedex, France

${ }^{4}$ Insitute of Nuclear Physics PAN, Kraków, Poland

${ }^{5}$ Texas A\&M University, Cyclotron Institute, College Station, TX, USA ${ }^{6}$ Helmholtzzentrum für Schwerionenforschung, Darmstadt, Germany ${ }^{7}$ CIMAP, ENSICAEN, CEA-CNRS/IN2P3, Caen, France ${ }^{8}$ CEA, LIST, LCAE, F-91191 Gif-sur-Yvette Cedex, France ${ }^{9}$ National Institute for Physics and Nuclear Engineering, RO-077125 Bucharest-Măgurele, Romania ${ }^{10}$ CRISMAT, ENSICAEN, CNRS/IN2P3, Caen, France
}

\begin{abstract}
Identification methods for photons, neutrons and heavy ions by using scintillator and semiconductor detectors are discussed by stressing the advantages of signal digitization.
\end{abstract}

The detector arrays including a large number of individual cells, e.g. [1,2], used in nuclear physics experiments, require automatic procedures to identify the reaction products. This is accomplished starting from the signals 
induced in different detection materials like organic scintillators for neutrons and photons, and inorganic scintillators, diamond or silicon, for charged particles. The description of their response functions has to remain simple and accurate in the same time. One must take into account the involved nonlinear processes like "quenching", electric carrier recombination and plasma delayed collection. The digitization of the signal, giving access to its shape as a function of time, increases the performance and opens new perspectives.

\section{Neutron identification in liquid scintillators}

The organic liquid scintillators like BC501A (the former NE213), available in big volume cells, are based on the molecular fluorescence induced at the passage of energetic photons, muons or neutrons. The emission of light accompanying the relaxation between singlet states is the fuorescence $\left(\approx 10^{-9} \mathrm{~s}\right.$ in liquid scintillators) while phosphorescence $\left(>10^{-6} \mathrm{~s}\right)$ means the radiative de-excitation, of low probability, from a triplet to the singlet ground state. Between, delayed fluorescence (10 to 100 times slower than fluorescence) also occurs at high densities of molecules excited in triplet states [3].

Photons and muons interacting with scintillator materials often transfer part of their energy to fast electrons which, due to their rather low stopping power, produce a weak density of excited molecules, decaying in turn by fluorescence. Neutrons transmit their energy to the host hydrogen nuclei characterized by a high electronic stopping power. Mainly via $\pi$-electron ionization followed by recombination, these protons cause a high density of excited molecules decaying by fluorescence, delayed fluorescence or phosphorescence [3]. Consequently, the scintillation signal following the interaction of a neutron, will contain, besides the "prompt" decay associated to fluorescence, a slow component, especially connected to delayed fluorescence. As for the slow phosphorescence, although present, it is not exploited at the high rate of events recorded in nuclear physics experiments. It is this difference of response to protons and fast electrons that makes possible the neutron/gamma $(n-\gamma)$ discrimination by pulse shape analysis. An example is shown in Fig. 1 for photon- and neutron-induced scintillations in a BC501A cell. The signals, normalized to the same total charge (related to the transferred energy), were averaged in several charge slices covering the light yield range 200 - 3000 keVee (see below). The associated photomultiplier signals were digitized by means of the Fast Acquisition SysTem for nuclEar Research (FASTER) developped at LPC Caen. While the $\gamma$-induced signals have identical shapes, those excited by neutrons show a larger slow fraction as the energy transferred to protons decreases. Additionally, the al- 


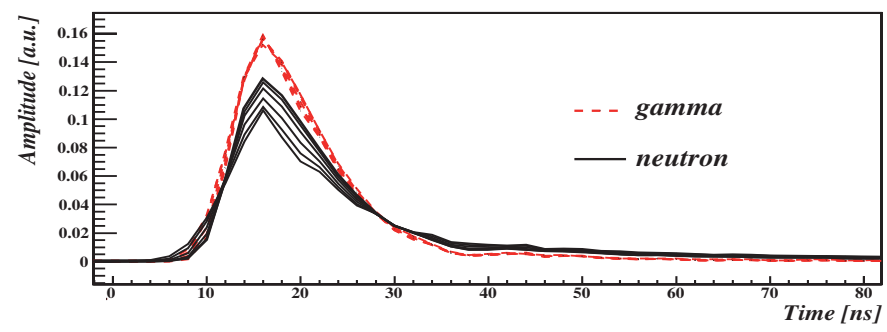

Figure 1: Average signals induced in a BC501A cell by neutrons and $\gamma$-rays.

most unique crossing point on the falling part (at around $30 \mathrm{~ns}$ ) suggests the position of a favourable border between two time gates of current integration giving $Q_{f}$ (fast) and $Q_{s}$ (slow) - two adequate fractions of the total charge $Q_{t}$. Several analysis algorithms have been imagined [4], recently based on digital acquisition [5]. The traditional method is based on the $Q_{s}-Q_{t}$ correlation, as in the two-dimensional plot obtained with standard VME analog electronics shown in Fig. 2, left panel. The muons from the electromagnetic showers induced in the Earth's atmosphere by high energy cosmic rays are placed in the same geometric locus as the $\gamma$-rays, but at higher $Q_{t}$. By
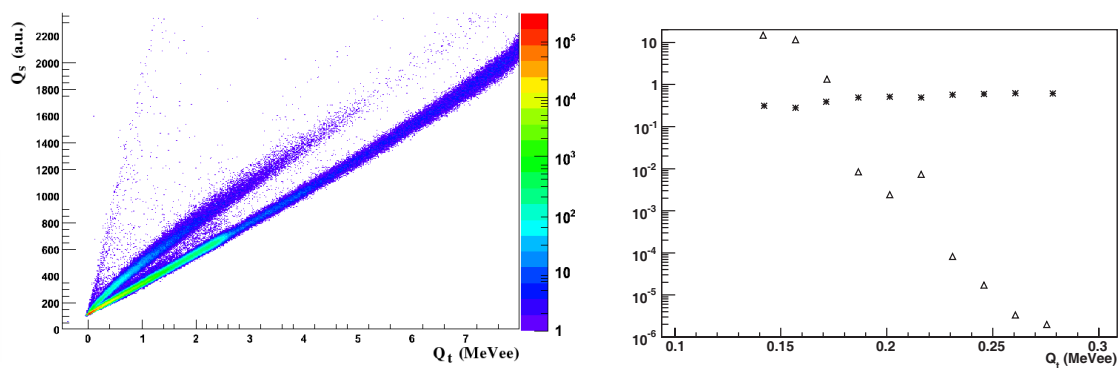

Figure 2: Left: traditional two-dimensional plot $Q_{s}$ vs $Q_{t}$ (calibrated into MeVee) illustrating the separation of neutrons (upper branch) and $\gamma$-rays (lower branch) from an AmBe source; the energetic muons are localized in the latter branch, but at higher total charges $Q_{t}$. Right: figure of merit (stars) and neutron area contamination (in \%) by $\gamma$-events (triangles) at one tenth of the neutron maximum.

means of $\gamma$ sources with known energies, the total charge was calibrated into $\mathrm{MeV}$ electron equivalent (MeVee). The energy deposited in the cell by the mentioned muons is useful at higher $Q_{t}[1]$. A figure of merit $(F O M)$ is used to quantify the discrimination quality in a total charge slice $\Delta Q_{t}$. It is 
frequently calculated as the ratio of the distance between the ridge lines of the two populations in Fig. 2 and the sum of their widths at a given fraction of the neutron maximum, in the considerred $\Delta Q_{t}$ slice. The problem occurs at low transferred energies inducing low light yield and hence a small $Q_{t}$. Due to the associated large fluctuations, the two geometrical loci - of the neutrons (via the recoil protons) and of the photons (recoil electrons) merge. In this case, it may be important to estimate the overlap of $n$ - and $\gamma$-loci, in particular the contamination $(C O N)$ of the neutron geometrical locus by $\gamma$ events. Both quantities FOM and $C O N$ are obtained, in each abscissa slice of Fig. 2 (left panel), by fitting - e.g. with the sum of two gaussians -, the number of events in the slice as a function of the discriminating variable $Q_{s}$. Taking as reference level one tenth of the neutron maximum for example, one gets $F O M_{1 / 10}$ and the contamination $C O N$ (very sensitive to $Q_{t}$ ), plotted in Fig. 2, right panel. Together, these quantities are well reporting on the $n-\gamma$ discrimination capability of a detector. The p-Terphenyl single crystal scintillator allows a better discrimination, indeed, but the available size is still limited. New plastic scintillators offer a promising alternative [6].

The accuracy of the discrimination depends much on the correct determination of the base line and the start moment of the signal. These aspects were carefully treated in FASTER - a digital acquisition system allowing the transfer of a high flux of time stamped events. It is appropriate for organic scintillators (500 MHz/12 bits digitizer) and for inorganic scintillators and semiconductors $(125 \mathrm{MHz} / 14 \mathrm{bits})$. FASTER is provided with FPGA-implemented single channel algorithms like QDCs, ADCs, CFDs. By using the time stamped events, one may realize a TDC like function to get the time difference between start and stop signals. The two dimensional constant fraction discriminator allows to set thresholds in amplitude and time width for accepting a signal. The CFD zero crossing time, calculated by parabolic interpolation, has a numerical precision of $8 \mathrm{ps}$ and hence the actual time resolution is practically dictated by that of the detector, of the order of $1 \mathrm{~ns}$ for our liquid scintillator and the photomultiplier read-out. A dynamical base line may be measured in a time gate preceeding the signal, and restored after it. As the signal is digitized very close to the read-out, the code for calculating different quantities of interest is written in the dedicated FPGA, accelerating the data flux towards a flexible tree architecture, in which decisions are taken afterwards at different levels. Entire signal waveforms for an off-line analysis may also be stored at a rate of $\leq 100 \mathrm{kHz}$. This generic and modular system is easily extensible from a few to about one hundred acquisition channels. FASTER is a versatile system opened to small and mean scale experiments. We have recently used it to measure 
the neutron cross talk yield between two liquid cells. Neutrons of $1.5 \mathrm{MeV}$ were produced via the ${ }^{3} H(p, n)^{3} \mathrm{He}$ reaction by means of a proton pulsed beam characterized by a period of $400 \mathrm{~ns}$ and a bunch width of a few ns. The detectors were placed at two different distances and angles (both of them larger for the second cell) with respect to the target and the beam direction, respectively. The TDC like function of FASTER allowed the time of flight (TOF) measurement and hence the determination of the neutron energy. The left panel of Fig. 3 displays the discriminating variable $Q_{s} / Q_{t}$ as a function of the TOF for the nearest cell. The $\gamma$-induced events are
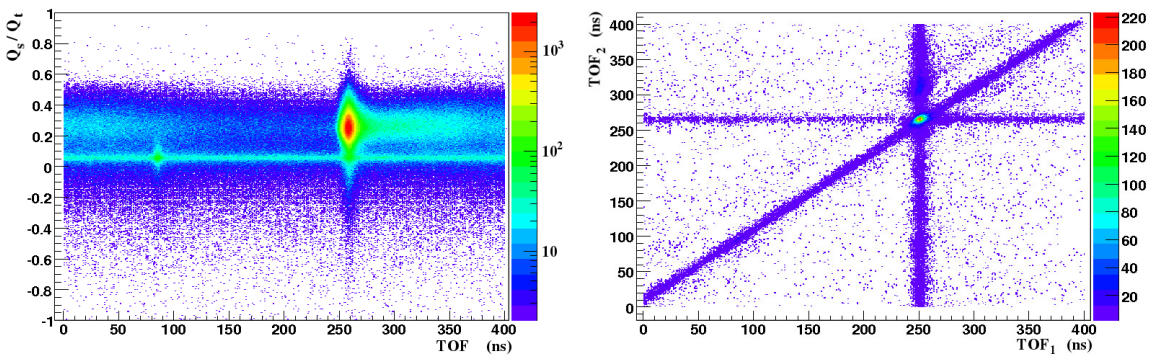

Figure 3: Left: ratio $Q_{s} / Q_{t}$ as a function of the time of flight. Right: Correlated events in two BC501A cells in terms of time of flight. See text for more detail.

localized in the lower, thin horizontal branch, and one may distinguish the direct $\gamma$ peak at $\approx 80 \mathrm{~ns}$. The $n$-induced events populate the upper branch, with a peak at $\approx 250 \mathrm{~ns}$ corresponding to the $1.5 \mathrm{MeV}$ neutrons and a long tail of slower neutrons. Below this neutron peak, in the $\gamma$-induced event branch, note also the neutrons viewed as $\gamma$-rays via the reaction $\left(n, n^{\prime} \gamma\right)$ on nuclei in the proximity of the cell. By simultaneousely looking at the TOF of the two scintillator cells - Fig. 3, right panel -, one may disentangle various correlations. The neutrons belonging to the main peak arrive in the first detector at $\approx 250 \mathrm{~ns}$, while in the second one, at $\approx 270 \mathrm{~ns}$. The probability to detect two neutrons from the same bunch is negligible. The vertical line corresponds to photons randomly arriving in the second detector in coincidence with the neutrons from the main neutron peak in the first detector, and viceversa for the horizontal line. The protuberence at $\approx 310 \mathrm{~ns}$ on the vertical line collect neutron cross talk events: neutrons detected and scattered in the first cell and arriving later on in the second one. The simulations will enlight us on the origin of the events located in the diagonal lines. The precise time calibration as well as the global analysis to determine efficien- 
cies and cross talk probabilities for various geometrical configurations and different low neutron energies $(\leq 4.9 \mathrm{MeV})$ are in progress [7].

\section{Response of $\mathrm{CsI}(\mathrm{Tl})$ scintillators to heavy ions}

Thallium-activated caesium iodide $(\mathrm{CsI}(\mathrm{Tl}))$ scintillating crystals offer the possibility of light charged particle isotopic identification by pulse shape discrimination technique. Their luminescence was quantified in a simple formalism [8] including quenching via electron-hole recombination inside the fiducial volume along the impinging particle path as well as high efficiency scintillation of the $\delta$-rays transporting the fraction $\mathcal{F}(x)$ of the electronic stopping power $S_{e}(x)$ outside this volume [9]. Under some assumptions [8], the rate of electron and hole local concentration $n(x, t)$ in this volume of high carrier density is driven, in a first approximation, by the equation:

$$
-\frac{d n(x, t)}{d t}=\left[\lambda_{A}+\lambda_{Q}\right] n(x, t)+\lambda_{R}[n(x, t)]^{2}
$$

with $\lambda_{A} \propto N_{A}$, the activator concentration (Tl centres), $\lambda_{Q} \propto\left(N_{D}+N_{n}\right)$, the concentration of "defects" due to thermal vibrations $N_{D}$ and to the interaction of the incident ion with the lattice nuclei $N_{n} \propto S_{n}(x)$, the nuclear stopping power; $\lambda_{R}$ stands for the electron-hole direct recombination scintillating in the UV band, not seen by the photomultiplier. The solution of eq. (1) and initial condition $n(x, 0)=(1-\mathcal{F}(x)) n_{0}(x), n_{0}(x) \propto S_{e}(x)$, is of the same type as the expression (13) in ref [10]. The infinitesimal light output $d L / d x$ is obtained by integrating over time the first term of eq. (1) and the total light output, by numerical integration over the total energy $E_{0}$ :

$$
\begin{array}{r}
L=a_{G}\left[\int_{0}^{E_{\delta}} \frac{1}{a_{R} S_{e}(E)} \ln \left(1-\frac{(1-\mathcal{F}(E)) a_{R} S_{e}(E)}{1+a_{n} S_{n}(E)+a_{R} S_{e}(E)}\right)^{-1} \frac{d E}{1+\frac{S_{n}(E)}{S_{e}(E)}}\right. \\
\left.+\int_{E_{\delta}}^{E_{0}} \frac{\mathcal{F}(E) d E}{1+\frac{S_{n}(E)}{S_{e}(E)}}\right] .
\end{array}
$$

It depends on four parameters $a_{G}, a_{n}, a_{R}$ and $E_{\delta}$ related respectively to the gain, nuclear and recombination quenching, and the energy threshold for the creation of $\delta$-rays. The corresponding formula (16) in ref. [8] was thus simplified without altering the quality of the light output description for various ions. The corresponding analytical expression was applied for heavy ion identification in $\mathrm{Si}-\mathrm{CsI}(\mathrm{Tl})$ telescopes [11].

The ordinary $\mathrm{Tl}$ doping of about $200 \mathrm{ppm}$, was optimized for $\gamma$-rays and light ions. In order to avoid the temporary depletion of the activator sites due to the high carrier density induced by heavy ions, one may increase, e.g. 
with one order of magnitude, the Tl doping. A new scintillation component (of time constant $\approx 100 \mathrm{~ns}$ ) is shown in the left panel of Fig. 4 for an $\approx 8 \mathrm{AMeV}{ }^{36} \mathrm{Cl}$ impinging nucleus. As the light efficiency increases, the energy resolution becomes better too. The temperature plays also a role in the light output efficiency as shown by Robertson et al. [12] for $14 \mathrm{MeV}$ protons and $5.3 \mathrm{MeV} \alpha$ particles. Their data are presented in the right panel of Fig. 4, suggesting that the optimum efficiency moves towards lower temperatures as the average electronic stopping power increases. New data concerning heavy ions would be highly interesting.
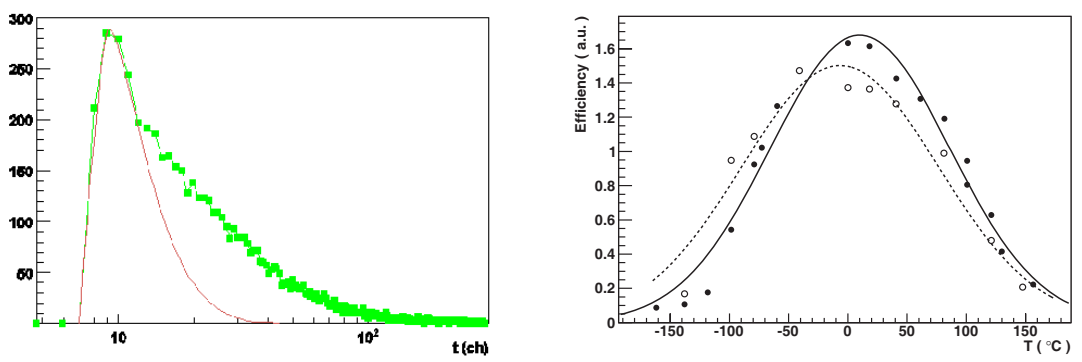

Figure 4: Left: light output induced by $\approx 8 \mathrm{AMeV}^{36} \mathrm{Cl}$ in a $2000 \mathrm{ppm} \mathrm{Tl}$ doped $\mathrm{CsI}(\mathrm{Tl})$ crystal (symbols connected by a thin line) vs time (4 ns/channel); the other curve stresses the fast peak due to the high Tl doping. Right: data from ref. [12]; the optimum efficiency temperature is lower for $\alpha$ particles (open circles and dashed curve) than for protons (full circles and solid curve).

\section{Diamond detector beam profiler}

Its high radiation hardness, high thermal conductivity and extreme mechanical strength recommend the Chemical Vapour Deposited (CVD) diamond as a promising material for nuclear physics purposes. Due to its high resistivity, the diamond endures large electric fields $(\approx 6 \mathrm{~V} / \mu \mathrm{m})$ in favour of a rapid collection of charge carriers created by ionizing particle. The dark current remains very low at room temperature $(<50 \mathrm{nA})$ because of the negligible intrinsic carrier concentration as result of the wide gap $(\approx 5.5 \mathrm{eV})$, allowing it to work as a simple (solid) ionization chamber. Due to its small relative permittivity $\epsilon_{r}=5.7$ and hence to a reduced capacitance in comparison with that of a silicon wafer of the same geometry, the current pulses induced in diamond have a very abrupt leading edge. The signals are shorter than those induced in $\mathrm{Si}$, due to the larger mobility of both types of carriers for diamond. The shortcomings are mainly related to the limited available 
area in case of single crystals (scCVD) and to the bulk polarization [13] for the polycrystalline (pcCVD) plates, englobing graphite inter-spaces acting as carrier traps; some palliatives exist to overcome this latter difficulty [14].

Characterization of the stable and radioactive beams throughout the future facilities - in particular the profile and the intensity - must be done with robust beam profilers. The rise time between $10 \%$ and $90 \%$ of the signal maximum delivered by two pcCVD detectors are shown Fig. 5, stressing the influence of the plate capacitance on the one hand, and that of the electronic chain, on the other hand. The left panel concerns a detector of $22 \times 22 \mathrm{~mm}^{2}$
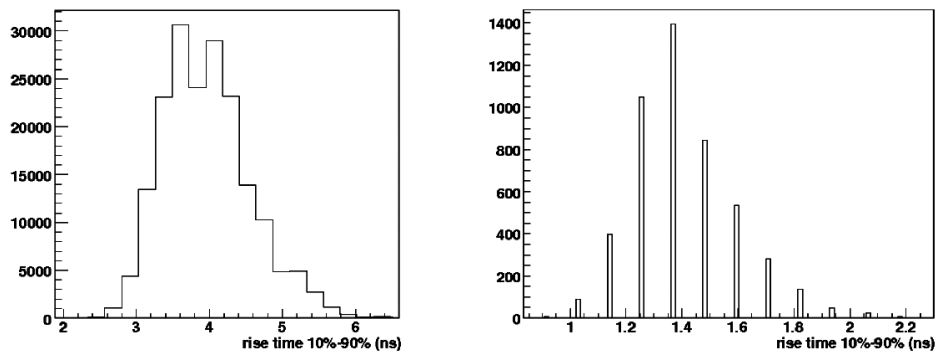

Figure 5: Comparison of the timing properties of a large area pcCVD detector (left panel) and a strip of small area (right panel). See text for more detail.

area and $\approx 300 \mu \mathrm{m}$ thickness $(\mathrm{C} \approx 80 \mathrm{pF}$ and time constant $\mathrm{RC} \approx 3.9 \mathrm{~ns})$ for $10.9 \mathrm{AMeV}^{58} \mathrm{Ni}$ ions accelerated on the SME line of GANIL. By adding the $0.9 \mathrm{~ns}$ contribution of the MATACQ - VME acquisition system (400 MHz band width (BW) and $2 \mathrm{GHz}$ sampling) one gets $\sqrt{3.9^{2}+0.9^{2}}=4 \mathrm{~ns}$ explaining the measured mean value. The histogram in the right panel was obtained with $13.7 \mathrm{AMeV}{ }^{16} \mathrm{O}$ from one pitch $\left(18 \mathrm{x} 1 \mathrm{~mm}^{2}\right)$ of a double-sided strip detector of $\approx 350 \mu \mathrm{m}$ thickness $(\mathrm{C} \approx 3 \mathrm{pF}$, time constant $\approx 0.13 \mathrm{~ns})$ by means of the same MATACQ card. The main contribution in the measured mean value comes in this case from the low BW of the MATACQ system. Such segmented detectors may be manufactured in $50 \times 50 \mathrm{~mm}^{2}$ area plates, completely adapted to get the profile of $10^{6}$ pps beams [14].

\section{Isotope identification in silicon detectors}

Selected high resistivity silicon detectors give acces to very good heavy ion identification results in $\Delta E-E$ telescopes [15], provided that an adequate method be applied. Eq. (1) keeps its validity in case of a silicon detector, the active role to collect the carriers being played by the electric field. The lattice defects dissociate also the carriers without trapping them and the signal is 


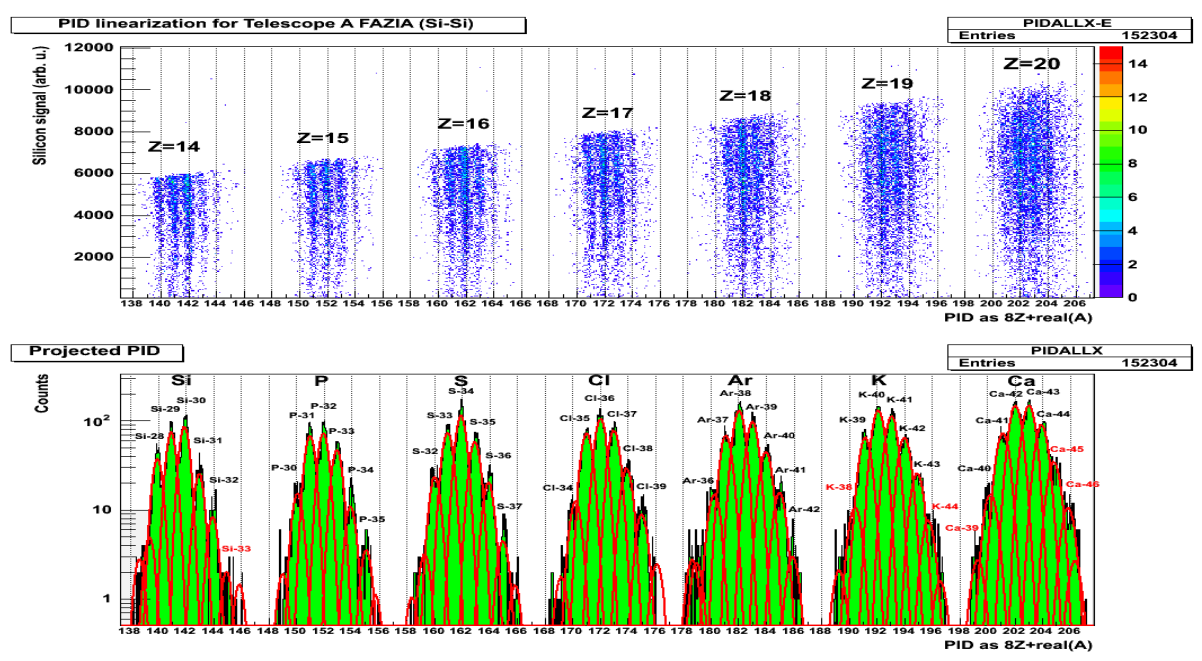

Figure 6: Linearization and isotopic identification in a $\Delta E-E$ silicon telescope.

associated to the first two terms. The direct electron-hole recombination is mainly responsible for the eventual pulse shape defect. The signal expression in each of the two detectors of the telescope is thus close to the eq. (2), the four parameters being determined by a global fit of the geometrical loci coresponding to a wide range of ions in a $\Delta E-E$ map. The good isotope identification is evidenced in the two-dimensional upper plot of Fig. 6 (for fixed $a_{n}=0$ ). As the linearization is not yet perfect, the quality is artificially degraded after the projection on the abscissa, presented in a logarithmic scale in the lower panel. This method allows to assign realistic confidence weights for each isotope when the mass separation is not $100 \%$.

The $\Delta E-E$ method implies a threshold due to the $\Delta E$ detector thickness. Conversely, there is no threshold, at least in principle, for the access to the shape of the digitized signal as a function of time [16]. This shape depends, via the plasma delay phenomenon, on the specific electronic stopping power and hence on the nature of the impinging ion. Heavy-ion-induced signals were recently described within a microscopic treatment of screening [17], and by a phenomenological approach based on the dielectric polarization and the progressive dissociation of the carrier pairs $[18,19]$, providing bases to address the funneling effect too [20].

High quality neutron and heavy ion identification is mandatory for nuclear physics experimental investigations and the limits have already 
been pushed very far. Promising efforts are made now in direction of large volume scintillating materials with new properties, large area scCVD diamond plates and selection of homogeneous silicon wafers - to be tested afterwards by physicists. The digital signal processing has opened new opportunities, eventually promoting new discriminating variables. Realistic simulations of the processes involved in the generation of the signal in various detectors are probably worthwhile investments. They may lead to appropriate "schematic" approximations which, combined with computing power, will hopefully provide improved automatic procedures of calibration and reaction product identification for large scale arrays in nuclear physics.

\section{References}

[1] I. Tilquin, Y. El Masri, M. Parlog et al., Nucl. Instr. and Meth. in Phys. Res. A 365 (1995) 446.

[2] J. Pouthas et al., INDRA collaboration, Nucl. Instr. and Meth. in Phys. Res. A 357 (1995) 418; 369 (1996) 222.

[3] J.B. Birks, in The theory and practice of scintillation counting, edited by Pergamon Press (1964).

[4] P.A. Söderström et al., Nucl. Instr. Meth. Phys. Res. A 594 (2008) 79.

[5] C. Guerrero et al., Nucl. Instr. Meth. in Phys. Res. A 597 (2008) 212.

[6] N. Zaitseva et al., Nucl. Instr. Meth. in Phys. Res. A 668 (2012) 88.

[7] M. Sénoville, Thèse de doctorat de l'Université de Caen, in progress.

[8] M. Pârlog et al., INDRA collaboration, Nucl. Instr. and Meth. in Phys. Res. A 482 (2002) 674; 482 (2002) 693.

[9] A. Meyer et al., Phys. Rev. 128 (1962) 98 and references therein.

[10] G. Tăbăcaru, B. Borderie, A. Ouatizerga, M. Pârlog et al., INDRA collaboration, Nucl. Instr. and Meth. in Phys. Res. A 428 (1999) 379.

[11] O. Lopez and M. Pârlog, INDRA collaboration, Proc. Intl. Workshop on Multifragmentation IWM2009, Catania (Italy) (2009) 148.

[12] J.C. Robertson et al., Proc. Phys. Soc. 78 (1961B) 1188.

[13] E.K. Souw et al., Nucl. Instr. Meth. in Phys. Res. A 400 (1997) 69. 
[14] M. Pârlog et al., http://www-norhdia.gsi.de/CARAT01/CARAT01Talks/

[15] FAZIA project, http://fazia.in2p3.fr

[16] S. Carboni et al., FAZIA collaboration, Nucl. Instr. Meth. in Phys. Res. A 664 (2012) 251;

[17] Z. Sosin, arXiv:1201.2188v1 [nucl-th]

[18] M. Pârlog, H. Hamrita, B. Borderie, FAZIA collaboration, Nucl. Instr. and Meth. in Phys. Res. A 613 (2010) 290.

[19] H. Hamrita, M. Pârlog, B. Borderie et al., FAZIA collaboration, Nucl. Instr. Meth. in Phys. Res. A 642 (2011) 59.

[20] C.M. Hsieh, P.C. Murley and R.R. O'Brien, IEEE Trans. Electron Dev. EDL-2 (1981) 103; ED-30 (1983) 686. 